\title{
In vitro characterization of human bone marrow mesenchymal stem cell-derived motor neurons induced by epigenetic modifiers
}

\author{
Davood Sanooghi ${ }^{1}$, Parham Vahdani ${ }^{2}$, Zohreh Bagher ${ }^{3}$, Faezeh Faghihi ${ }^{4^{*}}$ and Abolfazl Lotfi ${ }^{5}$
}

\begin{abstract}
Background: Motor neurons (MNs) are distinct types of cells in the dorso-ventral axis of the spinal cord. These cells are developed in the presence of two main morphogens, including Sonic hedgehog (Shh) and retinoic acid (RA). On the other hand, human bone marrow mesenchymal stem cells (hBM-MSCs) are known as a multipotent type of cells with neural differentiation capacity. In this regard, the aim of this study was to quantitatively evaluate the expression of $\mathrm{MN}$-related genes and the potent epigenetic regulatory genes involved in neurogenesis, including Enhancer of zeste homolog 2 (EZH-2) and P300, during hBM-MSC differentiation into MN-like cells, using RA and Shh. After isolating and inducing the cells with Shh and RA, the results were evaluated using immunocytochemistry and qRT-PCR.
\end{abstract}

Results: Our findings showed that the treated cells could express choline acetyltransferase (ChAT) and insulin gene enhancer binding protein-1 (Islet-1) antigens at the protein level, 2 weeks after induction. Moreover, at the second week after induction, the induced cells expressed MN-related genes (ChAT and ISLET-1) and epigenetic regulatory genes (EZH-2 and P300) at significant levels compared to the control (non-treated BM-MSCs) and to the induced cells at the first week (day 7). In addition, the expression of $E Z H-2$, as a histone-modifying gene, was also significantly upregulated at the first week compared to the control. No significant upregulation was detected in the expression of motor neuron and pancreas homeobox $1(M N X-1)$ in the treated groups compared to the control group.

Conclusion: We concluded that epigenetic modifiers, P300 and EZH-2, are important mediators for regulating the process of motor neuron differentiation induced by RA and Shh.

Keywords: Motor neuron, Bone marrow, Mesenchymal stem cell, Epigenetic, P300, EZH-2

\section{Background}

Motor neurons (MNs) are known as a distinct type of cells with defined functions along the dorso-ventral axis of the spinal cord. Two main morphogens, including retinoic acid (RA) and Sonic hedgehog (Shh), make role in the developmental specification of these cells [1]. Motor neuron-related diseases threaten the lives of many

\footnotetext{
*Correspondence: faghihif@iums.ac.ir; faezefaghihi@yahoo.com

${ }^{4}$ Cellular and Molecular Research Center, Iran University of Medical Sciences, Tehran, Iran

Full list of author information is available at the end of the article
}

patients throughout the world. Since there is no efficient clinical treatment for these patients, medical interventions such as cell therapy still bring hope to these people. Human bone marrow mesenchymal stem cells (hBM-MSCs) are multipotent cells characterized by selfrenewal and immunomodulatory properties $[2,3]$. Cell fate of MSCs would be changed depending on the source of origin and by the ingrained epigenetic memory signatures of the cells [4]. Synergistic administration of RA and Shh leads to the expression of motor neuronrelated markers in cultured stem cells [5]. Retinoic acid 
plays a major role in the development of stem cells; however, little is known about the mechanisms for the repression of RA-regulated genes [6]. Moreover, the evolutionary conserved developmental morphogens, the Hedgehog family, have critical roles in stem cell development and functions [7]. During the development of human stem cells, differential gene expression is retained through mitosis. Such stable alterations in the gene expression occur during progression from stem cells into differentiated progenies. The commitment of stem cells to differentiate into a particular cell type requires upregulating the expression of genes responsible for a specific phenotype and suppressing the expression of other genes that maintain the stemness properties $[8,9]$. By imposing functionally relevant modifications to the genome, histone-modifying enzymes regulate the accessibility of transcription factors and other modulators to the genes $[9,10]$. For example, a histone-lysine $N$-methyltransferase enzyme called enhancer of zeste homolog 2 (EZH-2) regulates the transition from proliferation to differentiation and accelerates the onset of neurogenesis [11, 12]. Moreover, it regulates the balance between self-renewal and differentiation in the cerebral cortex cells [11], controls neural stem cell state [13], and prevents premature differentiation [11, 14]. Through an epigenetic mechanism, Shh signaling regulates the expression of EZH-2 and manages the expression of genes involved in numerous physiological functions such as neural cell survival and differentiation, in vivo $[15,16]$.

On the other hand, a histone acetyltransferase, P300, makes a role in the regulation of neurogenesis in the spinal cord motor neurons $[5,17]$. Tight control on the function of P300 is critical to ensure precise histone acetylation and gene activation. Numerous studies have examined the functional requirement of P300 to act as a co-activator or an acetyltransferase for other transcription regulators [18]. In embryonic spinal motor neurons, P300 and CREB-binding protein (CBP) contribute to the maintenance of Islet-1 expression as the main motor neuron-related transcription factor [19]. The decrease in the number of $\mathrm{MNs}$ in CBP/P300 compound mutant embryos confirms that these coactivators act redundantly to promote MN specification, in vivo.

The gene expression and epigenetic regulation underlying the process of mesenchymal stem cell differentiation into motor neurons are poorly understood. Therefore, the purpose of this study was to quantitatively investigate the expression of motor neuron-related genes and the potent epigenetic regulatory genes involved in neurogenesis, including EZH2 and P300, during differentiation of human BM-MSCs into motor neuron-like cells, using RA and Shh.

\section{Methods \\ Isolation and culture of human bone marrow-derived mesenchymal stem cells}

The protocol for the aspiration of human bone marrow was approved by the university research ethics committee. After diluting $5 \mathrm{ml}$ of the aspirated samples with an equal volume of phosphate-buffered saline (PBS; Gibco, USA), to isolate bone marrow mesenchymal stem cells (BM-MSCs), the solution was loaded onto $20 \mathrm{ml}$ of Ficoll (Inno-Train, Germany) and centrifuged $(400 \times g, 20$ $\mathrm{min})$. The mononuclear cells were then collected, washed twice with PBS, and seeded in tissue culture flasks $75 \mathrm{~cm}^{2}$ (TPP, Switzerland) at a density of $10^{5}$ cells/ml using Dulbecco's modified Eagle's medium (DMEM-F12) supplemented with $15 \%$ fetal bovine serum (FBS), $100 \mathrm{U} / \mathrm{ml}$ penicillin, and $100 \mu \mathrm{g} / \mathrm{ml}$ streptomycin (all reagents from Gibco, USA). The cells were cultured at $37{ }^{\circ} \mathrm{C}$ in an incubator containing 5\% $\mathrm{CO}_{2}$. The culture medium was refreshed every 3 days. The cells were passaged at the ratio of $1: 3$, upon reaching $80 \%$ confluence [20]. The third passage cells were used for subsequent experiments.

\section{Characterization of mesenchymal stem cells}

Mesenchymal stem cells were characterized using mono-color cytofluorimetric analysis, according to our previous protocol [21]. To do that, $1 \times 10^{5}$ of these cells with $10 \%$ goat serum were incubated at $4{ }^{\circ} \mathrm{C}$ for an hour. After removal of the serum, the cells were labeled with monoclonal antibodies conjugated with red phycoerythrin (PE) or green fluorescein isothiocyanate (FITC)-conjugated monoclonal antibodies against human antigens including CD34, CD44, CD45, CD73, and CD90 (BD bioscience) at $4{ }^{\circ} \mathrm{C}$ for $40 \mathrm{~min}$. In each case, an isotypematched control was used. Antigen expression data obtained by FACSCalibur Flow Cytometer (Becton Dickinson, UK) were analyzed by the FlowJo software.

\section{Induction of mesenchymal stem cells into motor neuron- like cells}

Human bone marrow mesenchymal stem cells were induced into motor neuron-like cells based on our previous protocol [20, 21]. In summary, the cells were cultured overnight at a density of $10^{5}$ cells/well in a 24well plate containing a complete expansion medium at $37{ }^{\circ} \mathrm{C}$ and $5 \% \mathrm{CO}_{2}$ humid incubator. Afterward, the existing medium was replaced with a pre-inductive medium consisting of DMEM-F12, 20\% FBS, $10 \mathrm{ng} / \mathrm{ml}$ bFGF (Sigma, USA), $250 \mathrm{mM}$ isobutylmethylxanthine (IBMX; Gibco, USA), $100 \mathrm{mM} \beta$-mercaptoethanol $(\beta$ ME; Gibco, USA), and 2\% B27 (Invitrogen, USA) and stored overnight in an incubator. On the following day, after removing the inductive medium, the induced cells were treated with the first differentiation medium 
including DMEM-F12 supplemented with $0.01 \mathrm{mM}$ RA (Sigma, USA), $100 \mathrm{ng} / \mathrm{ml} \mathrm{Shh} \mathrm{(R \& D,} \mathrm{USA),} \mathrm{and} \mathrm{0.2 \%}$ B27 for a week. The first differentiation medium was then substituted with the second differentiation medium containing DMEM-F12 with brain-derived neurotrophic factor (BDNF; Invitrogen, USA) and 0.2\% B27 as surviving factors, and differentiating cells were cultured in this medium for 1 week later.

\section{Immunocytochemical staining}

To evaluate the expression of relevant antigens at the protein level, the treated cells were fixed using $4 \%$ paraformaldehyde. Then, the cells were permeabilized by treatment with $0.2 \%$ Triton X-100 (Gibco, USA) in PBS. After washing, they were incubated with diluted $10 \%$ goat serum in PBS as a blocking buffer at room temperature for an hour to prevent nonspecific interactions. Thereafter, primary antibodies against human choline acetyltransferase (ChAT; Abcam, USA), Islet-1 (Santa Cruz, USA), and Mnx-1 (Abcam, USA) were incubated overnight with samples at 4 ${ }^{\circ} \mathrm{C}$. After washing with PBS, the cells were labeled by incubation with the corresponding secondary antibodies (Sigma, USA) conjugated to FITC or PE at $37^{\circ} \mathrm{C}$ for $45 \mathrm{~min}$. Eventually, DAPI (Sigma, USA) was used to stain the nuclei, and
Table 1 List of primers used in qRT-PCR

\begin{tabular}{ll}
\hline Target gene & Primer sequence \\
\hline GAPDH & F: CTCATTCCTGGTATGACAAC \\
& R: CTTCCTCTTGTCTCTTGCT \\
CHAT & F: GCA GGAGAAGACAGCCAACT \\
& R: TGCAAACCTCAGCTGGTCAT \\
MNX-1 & F: AGCACCAGTTCAAGCTCAACA \\
& R: ACCAAATCTTCACCTGGGTCTC \\
ISLET-1 & F: ATATCAGGTTGTACGGGATCAAATG \\
P300 & F: CACGCATCACGAAGTCGTTC \\
& R: GCAGAGGATTCATGTTCTGCAAG \\
EZH-2 & F: CCGGGCTAGGTTAATTGGGACCAAA \\
& R: CTCGAGTTTGTCCCAATAACCT \\
\hline
\end{tabular}

cell observation was accomplished by a DP70 fluorescence microscope (Olympus, Japan).

\section{Q-RT PCR}

The expressions of motor neuron-associated antigens and the regulating histone acetyltransferase and histone methyltransferase genes were confirmed by real-time PCR. After cell harvesting at days 7 and 14 post-induction, total RNA was extracted by TRIzol Reagent (Sigma, Germany). Then,
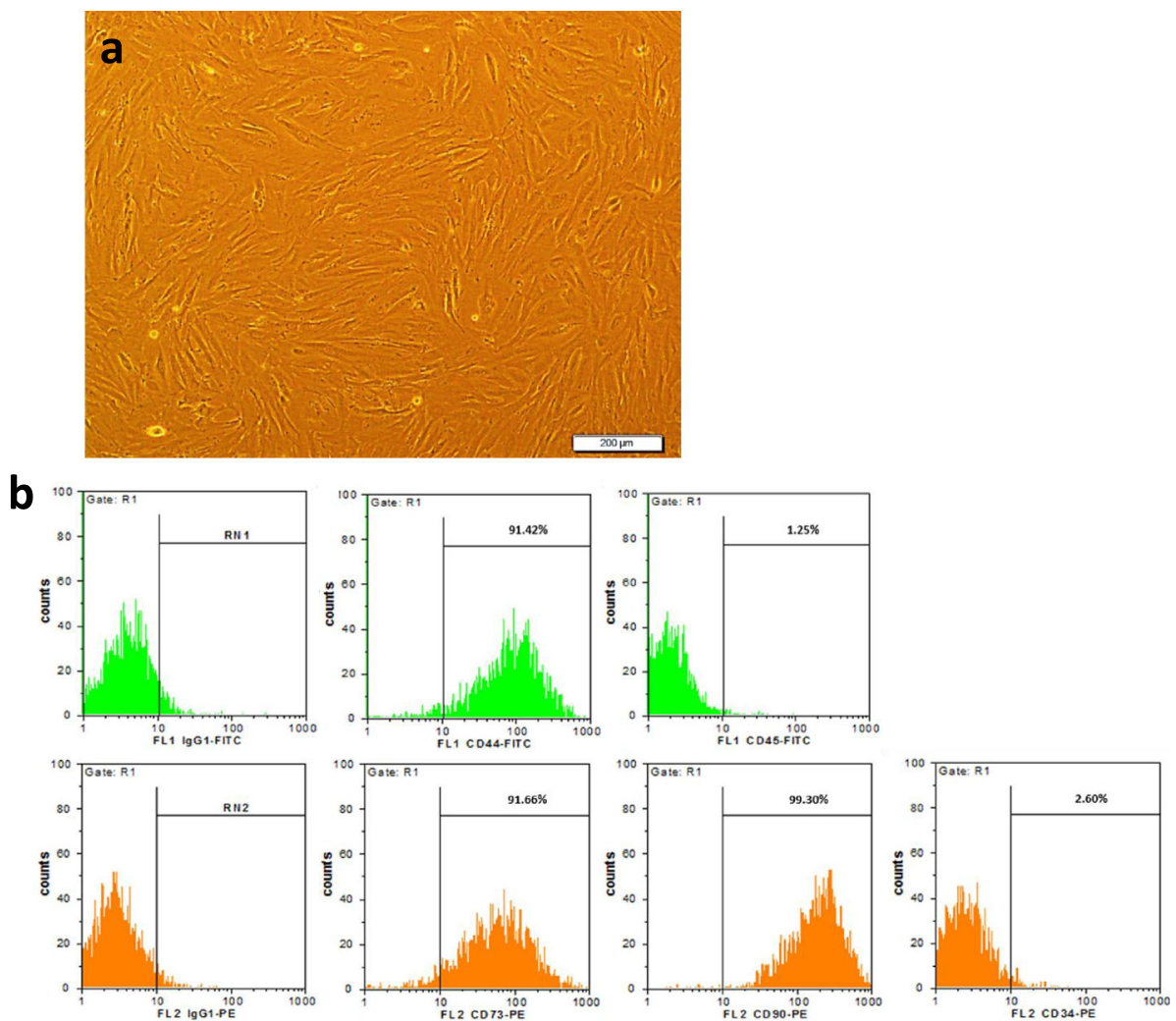

Fig. 1 Isolation and characterization of human bone marrow-derived mesenchymal stem cells. a BM-MSCs had fibroblast-like morphology after three passages (magnification $\times 4$ ). b Cytofluorimetric analysis of the isolated cells at passage 3 revealed that they expressed CD44, CD73, and CD90 antigens, but they could not express hematopoietic and leukocyte markers, including CD34 and CD45 
the purity of RNA was quantitatively evaluated by spectrophotometer, and cDNA was synthesized from the extracted RNA using a cDNA Synthesis Kit (Fermentase, Canada). Eventually, RT-PCR reactions were performed in a 7500 real-time PCR system (Applied Biosystems, USA) in such a way that in each reaction, $2 \mu \mathrm{l}$ of $12.5 \mathrm{ng}$ cDNA was mixed with $5 \mu \mathrm{l}$ of SYBR Green Master Mix (Applied Biosystems, USA) and $1 \mu \mathrm{l}$ of corresponding forward and reverse primers (Table 1), and the total volume was reached to 20 $\mu \mathrm{l}$ by adding double-distilled water. The expression level of the GAPDH gene was considered as an internal control to normalize the expression levels of selected genes. Each target gene expression was evaluated in duplicate for three different mRNA samples.

\section{Results}

Isolation and characterization of human bone marrow mesenchymal stem cells

The isolated MSCs taken from human bone marrow had spindle-like morphology at passage 3 (Fig. 1a). These cells were able to express CD44, CD73, and CD90 markers on their surfaces, but they could not express hematopoietic antigens, such as CD45 and CD34 (Fig. 1b).
Differentiation of bone marrow mesenchymal stem cells into motor neuron-like cells

The expression of motor neuron-related markers, including ChAT, Islet-1, and Mnx-1, were evaluated using immunocytochemical staining. The results showed that the cells could express ChAT $(45 \pm 13.4 \%)$ and Islet-1 (53 $\pm 10.1 \%)$. No expression of Mnx-1 was detected on day 14 (Fig. 2).

\section{Evaluation of expression by real-time PCR}

Treatment of hBM-MSCs with the induction medium led to the upregulation of motor neuron-related genes, including ISLET-1 and ChAT as well as histonemodifying enzyme genes, $P 300$ and $E Z H-2$, at the second week of induction when RA and Shh were removed $(p \leq 0.05)$. Moreover, significant upregulation of $E Z H-2$ was also detected at the first week when the result was compared with non-treated BM-MSCs as the control $(p \leq 0.05)$. We could not detect any upregulation in the expression of $M N X-1$ compared to the control (Fig. 3).

\section{Discussion}

Mesenchymal stem cells derived from bone marrow are known as multipotent cells with immunomodulatory properties which have the potential to differentiate into

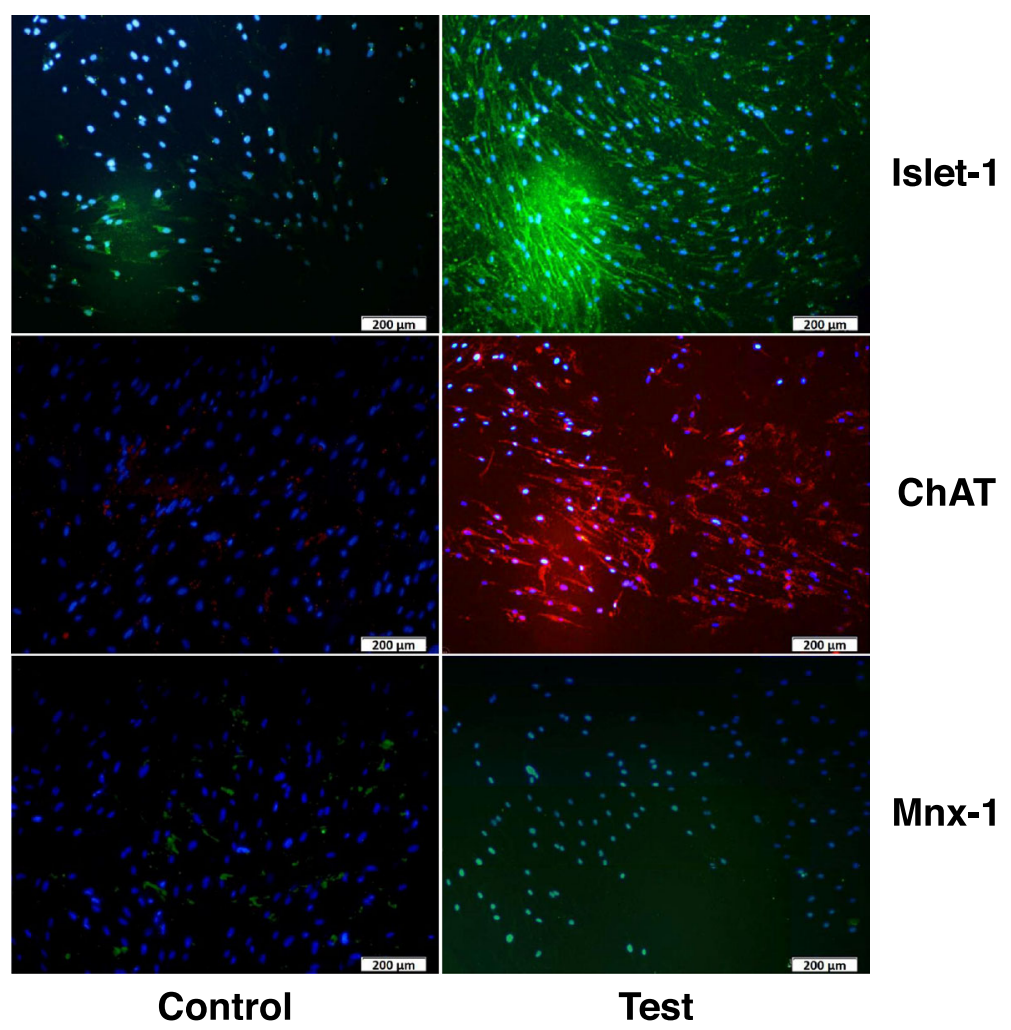

Fig. 2 Immunostaining of motor neuron-like cells derived from hBM-MSCs. The expression of Islet-1 (53 \pm 10.1\%), ChAT (45 $\pm 13.4 \%)$, and Mnx-1 antigens were investigated at the second week post-induction in the test group (treated hBM-MSCs) compared with the control group (non-treated hBM-MSCs) 


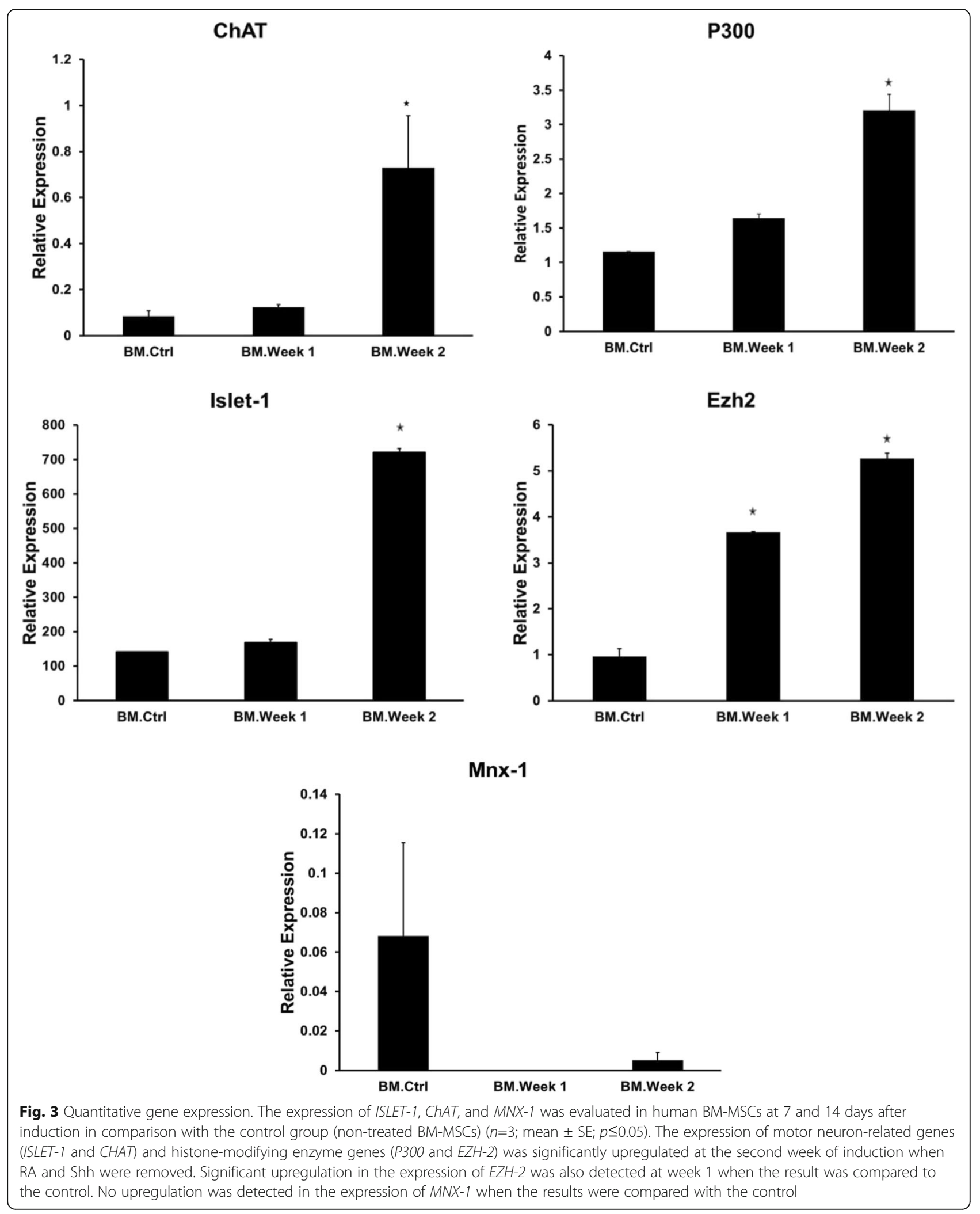


neural cell lineages. During stem cell differentiation, some specific genes associated with the development of a particular phenotype are upregulated, and the genes accountable for stemness characteristics are inhibited [8, $22]$. For example, in histone-mediated epigenetic regulation of cell differentiation, some modifications alter the access of transcription factors and other modulators to gene promoters $[9,10]$.

In the process of motor neuron differentiation from mesenchymal stem cells, the relationship between gene expression and epigenetic regulation is poorly understood. So, the aim of this study was to quantitatively investigate the expression of the candid genes associated with motor neuron differentiation along with two potent epigenetic regulatory genes, called EZH-2 and P3OO, in motor neuron-like cells derived from hBM-MSCs. To induce the differentiation, we used two morphogens called RA and Shh which are responsible for the dorso-ventral specification of the spinal cord and the development of motor neurons, in vivo. To our knowledge, this is one of the first studies to evaluate the expression of $E Z H-2$ and P300 during motor neuron differentiation of hBMMSCs.

According to our results, the treated cells could express ChAT and Islet-1 as motor neuron-specific markers at both mRNA and protein levels at the second week of induction. Data were confirmed by qRT-PCR and immunocytochemistry. It has already been approved that the synergistic administration of RA and Shh upregulates the expression of Islet-1 and ChAT [23]. Islet-1 as a LIM-homeodomain transcription factor is involved in the development of cholinergic amacrine cells and differentiation of motor neurons [24]. We could not detect any upregulation in the expression of $M N X-1$ upon differentiation. Suppression of the expression of $M N X-1$ in mice can reduce the number of developing motor neurons; however, the target genes downstream of ISLET-1 are independent of $M N X-1$ [25]. We could detect significant upregulation in the expression of ISLET-1and $C h A T$ at the second week of the induction after removal of RA and Shh. Accordingly, it seems that the morphogens tend to keep the cells in an immature state.

We could also detect the expression of EZH-2 and P300 genes for histone-lysine $N$-methyltransferase and histone acetyltransferase enzymes, respectively. EZH-2 is crucial for regulating the transition from proliferation to differentiation as well as suppressing forebrain traits [26]. It also controls the condition of neurogenesis by coordination between neuro-regeneration and differentiation in the cerebral cortex $[11,13]$. In this regard, it seems that the expression of $E Z H-2$ sets on the process of neurogenesis initiation by the transition from proliferation to differentiation and prevents premature differentiation in our experiment. It has been shown that $E Z H-2$ has a serious time-dependent role during neurogenesis [27]. In our study, the expression of EZH-2 upregulated in the presence of RA and Shh and continued to be expressed even after the removal of these two morphogens.

Based on our results, the expression of the P300 gene increased at the second week of differentiation after the removal of RA and Shh. The expression of P300 is required for astrocyte development and axon regeneration $[14,28]$. The development of motor neurons in the spinal cord is dependent on CBP [29]. Through synergistic interaction with RA receptor (RAR) and Neurogenin 2, CBP and its paralog, P300, as co-activator are involved in regulating neurogenesis and following stages of neural differentiation in spinal motor neurons $[28,29]$. This coactivator participates in maintaining the expression of ISLET-1 in embryonic spinal MNs. As we could see, the expression of ISLET-1 was also upregulated at the second week post-induction. According to Toch et al., the mutation in the CBP/P300 compound reduces the number of developing MNs in the embryos. This indicates that P300 acts redundantly in neural progenitors to promote MN specification [19]. Interestingly, other researchers suggest that CBP and P300 also act in postmitotic MNs to regulate later aspects of development [29]. This phenotype is due to the downregulation of ISLET-1 in MNs, suggesting that the activity of CBP and P300 in post-mitotic MNs may depend on the downregulation of ISLET-1 [19].

\section{Conclusion}

It can be concluded that neuronal inducing morphogens, including RA and Shh, have regulatory effects on the expression of epigenetic regulatory genes, $E Z H-2$ and P300, during differentiation of mesenchymal stem cells into motor neurons. These regulatory effects eventually lead to the expression of motor neuron-related markers such as ChAT and ISLET-1, which indicate the completion of the differentiation process.

\section{Abbreviations \\ hBM-MSCs: Human bone marrow mesenchymal stem cells; MNs: Motor neurons; Shh: Sonic hedgehog; RA: Retinoic acid; EZH-2: Enhancer of zeste homolog 2; ChAT: Choline acetyltransferase; ISLET-1: Insulin gene enhancer binding protein-1; MNX-1: Motor neuron and pancreas homeobox 1; CBP: CREB-binding protein; IBMX: Isobutylmethylxanthine; $\beta-M E: ~ \beta-$ Mercaptoethanol; BDNF: Brain-derived neurotrophic factor}

\section{Acknowledgements}

Not applicable

\section{Authors' contributions}

DS and PV contributed to the investigation, data curation, and formal analysis. ZB contributed to the methodology. FF contributed to the supervision, conceptualization, and writing - review and editing. AL contributed to the conceptualization and writing - review and editing. All authors read and approved the final manuscript. 


\section{Funding}

This study was financially supported by grant no. 95-03-117-29420 from Iran University of Medical Sciences and grant no. 95835564 from Iran National Science Foundation (INSF). The funders had no role in the study design, data collection and analysis, decision to publish, or preparation of the manuscript.

\section{Availability of data and materials}

The datasets used and/or analyzed during the current study are available from the corresponding author on reasonable request.

\section{Declarations}

\section{Ethics approval and consent to participate}

Not applicable.

The protocol for aspiration of human bone marrow was approved by the Research Ethics Committee of Iran University of Medical Sciences (IR.IUMS.REC 1395.95-03-117-29420).

\section{Consent for publication}

Not applicable.

\section{Competing interests}

The authors declare that they have no competing interests.

\section{Author details}

${ }^{1}$ Cancer Research Center, Shahid Beheshti University of Medical Sciences, Tehran, Iran. ${ }^{2}$ Department of Neuroscience, Faculty of Advanced Technologies in Medicine, Iran University of Medical Sciences, Tehran, Iran. ${ }^{3}$ ENT and Head \& Neck Research Center and Department, The Five Senses Institute, Hazrat Rasoul Akram Hospital, Iran University of Medical Sciences, Tehran, Iran. ${ }^{4}$ Cellular and Molecular Research Center, Iran University of Medical Sciences, Tehran, Iran. ${ }^{5}$ Damavand Agricultural College, Technical and Vocational University, Tehran, Iran.

\section{Received: 29 October 2020 Accepted: 20 April 2021}

\section{Published online: 03 June 2021}

\section{References}

1. Mich JK, Chen JK (2011) Hedgehog and retinoic acid signaling cooperate to promote motoneurogenesis in zebrafish. Development. 138(23):5113-5119. https://doi.org/10.1242/dev.066225

2. Hermann A, Gastl R, Liebau S, Oana Popa M, Fiedler J, Boehm BO, Maisel M, Lerche H, Schwarz J, Brenner R, Storch A (2004) Efficient generation of neural stem cell-like cells from adult human bone marrow stromal cells. J Cell Sci. 117(19):4411-4422. https://doi.org/10.1242/jcs.01307

3. Kaplan JM, Youd ME, Lodie TA (2011) Immunomodulatory activity of mesenchymal stem cells. Curr Stem Cell Res Ther. 6(4):297-316. https://doi. org/10.2174/157488811797904353

4. Xu L, Liu Y, Sun Y, Wang B, Xiong Y, Lin W (2017) Tissue source determines the differentiation potentials of mesenchymal stem cells: a comparative study of human mesenchymal stem cells from bone marrow and adipose tissue. Stem Cell Res Ther. 8(1):275. https://doi.org/10.1186/s13287-017-0716$x$

5. Lee SK, Paff SL (2003) Synchronization of neurogenesis and motor neuron specification by direct coupling of bHLH and homeodomain transcription factors. Neuron. 38(5):731-745. https://doi.org/10.1016/S0896-6273(03)002 96-4

6. Urvalek AM, Guda LJ (2014) Retinoic acid and histone deacetylases regulate epigenetic changes in embryonic stem cells. J Biol Chem. 289(28):1951919530. https://doi.org/10.1074/jbc.M114.556555

7. Fattahi S, Pilehchian Langroudi M, Akhavan-Niaki H (2018) Hedgehog signaling pathway: epigenetic regulation and role in disease and cancer development. J Cell Physiol. 233(8):5726-5735. https://doi.org/10.1002/jcp.2 6506

8. Kouzarides T (2007) Chromatin modifications and their function. Cell. 128(4): 693-705. https://doi.org/10.1016/j.cell.2007.02.005

9. Vincent A, Van Seuningen I (2009) Epigenetics, stem cells and epithelial cell fate. Differentiation. 78(2-3):99-107. https://doi.org/10.1016/j.diff.2009.07.002

10. Qiu J (2006) Epigenetics: unfinished symphony. Nature. 441(7090):143-145. https://doi.org/10.1038/441143a
11. Pereira JD, Sansom SN, Smith J, Dobenecker MW, Tarakhovsky A, Livesey FJ (2010) Ezh2, the histone methyltransferase of PRC2, regulates the balance between self-renewal and differentiation in the cerebral cortex. Proc Natl Acad Sci. 107(36):15957-15962. https://doi.org/10.1073/pnas.1002530107

12. Banerjee D, Liu Z, Doo-Yi O, Cam M, Kim B-H, Thiele C (2017) Retinoic acid (RA) relieves $E Z H 2$-mediated epigenetic suppression in high-risk neuroblastoma (HR-NB), pp 3872-3872. https://doi.org/10.1158/1538-7445.A M2017-3872

13. Rai K, Jafri IF, Chidester S, James SR, Karpf AR, Cairns BR, Jones DA (2010) Dnmt3 and G9a cooperate for tissue-specific development in zebrafish. J Biol Chem 285(6):4110-4121. https://doi.org/10.1074/jbc.M109.073676 Epub 2009 Nov 29. PMID: 19946145; PMCID: PMC2823551

14. Lilja T, Heldring N, Hermanson O (2013) Like a rolling histone: epigenetic regulation of neural stem cells and brain development by factors controlling histone acetylation and methylation. Biochim Biophys Acta. 1830(2):2354-2360. https://doi.org/10.1016/j.bbagen.2012.08.011

15. Gan L, Yang Y, Li Q, Feng Y, Liu T, Guo W (2018) Epigenetic regulation of cancer progression by EZH2: from biological insights to therapeutic potential. Biomark Res 6:10 Published 2018 Mar 9. doi:10.1186/s40364-0180122-2

16. Rao RC, Tchedre KT, Malik MTA, Coleman N, Fang Y, Marquez VE, Chen DF (2010) Dynamic patterns of histone lysine methylation in the developing retina. Invest Ophthalmol Vis Sci 51(12):6784-6792. https://doi.org/10.1167/ iovs.09-4730

17. Partanen M, Motoyama J, Hui CC (1999) Developmentally regulated expression of the transcriptional cofactors/histone acetyltransferases CBP and p300 during mouse embryogenesis. Int J Dev Biol. 43(6):487-494

18. Chen J, Li Q (2011) Life and death of transcriptional co-activator p300. Epigenetics. 6(8):957-961. https://doi.org/10.4161/epi.6.8.16065

19. Toch M, Clotman F (2019) CBP and p300 coactivators contribute to the maintenance of Is|1 expression by the Onecut transcription factors in embryonic spinal motor neurons. Mol Cell Neurosci. 101:103411. https://doi. org/10.1016/j.mcn.2019.103411

20. Faghihi F, Mirzaei E, Sarveazad A, Ai J, Barough SE, Lotfi A, Joghataei MT (2015) Differentiation potential of human bone marrow mesenchymal stem cells into motorneuron-like cells on electrospun gelatin membrane. J Mol Neurosci 55(4):845-853. https://doi.org/10.1007/s12031-014-0437-x

21. Yousefi B, Sanooghi D, Faghihi F, Joghataei MT, Latifi N (2017) Evaluation of motor neuron differentiation potential of human umbilical cord bloodderived mesenchymal stem cells, in vitro. J Chem Neuroanat. 81:18-26. https://doi.org/10.1016/j.jchemneu.2017.01.003

22. Jaenisch R, Bird A (2003) Epigenetic regulation of gene expression: how the genome integrates intrinsic and environmental signals. Nat Genet. 33(S3): 245-254. https://doi.org/10.1038/ng1089

23. Park H, Park W, Cho JS, Park CK, Jung SJ, Park CH (2012) Directed induction of functional motor neuron-like cells from genetically engineered human mesenchymal stem cells. Plos One 7(4):e35244

24. Galli-Resta L, Resta G, Tan SS, Reese BE (1997) Mosaics of islet-1-expressing amacrine cells assembled by short-range cellular interactions. J Neurosci. 17(20):7831-7838. https://doi.org/10.1523/JNEUROSCI.17-20-07831.1997

25. Liang I, Song MR, Xu ZG, Lanuza GM, Liu Y, Zhuang T, Chen Y, Pfaff SL, Evans SM, Sun $Y(2011)|s| 1$ is required for multiple aspects of motor neuron development. Mol Cell Neurosci. 47(3):215-222. https:/doi.org/10.1016/j.mcn.2011.04.007

26. Zemke M, Draganova K, Klug A (2015) Loss of Ezh2 promotes a midbrain-toforebrain identity switch by direct gene derepression and Wnt-dependent regulation. BMC Biol. 13(1):103. https://doi.org/10.1186/s12915-015-0210-9

27. O'Carroll D et al (2001) The polycomb-group gene Ezh2 is required for early mouse development. Mol Cell Biol. 21(13):4330-4336. https://doi.org/10.112 8/MCB.21.13.4330-4336.2001

28. Cheng PY, Lin YP, Chen YL, Lee YC, Tai CC, Wang YT, Chen YJ, Kao CF, Yu J (2011) Interplay between SIN3A and STAT3 mediates chromatin conformational changes and GFAP expression during cellular differentiation. Plos One. 6(7):e22018. https://doi.org/10.1371/journal.pone.0022018

29. Lee S, Lee B, Lee JW, Lee SK (2009) Retinoid signaling and neurogenin2 function are coupled for the specification of spinal motor neurons through a chromatin modifier CBP. Neuron. 62(5):641-654. https://doi.org/10.1016/j. neuron.2009.04.025

\section{Publisher's Note}

Springer Nature remains neutral with regard to jurisdictional claims in published maps and institutional affiliations. 